\title{
Acute coronary syndrome without ST-elevation in hypertrophic cardiomyopathy and normal coronary angiogram: a case report
}

\author{
Snezana Lazic*, Slavica Pajovic, Radojica Stolic, Daniela Celic \\ Faculty of Medical Science University of Pristina, Kosovska Mitrovica, Kosovo
}

Objectives: The existence of myocardial infarction despite normal coronary arteries was recognized several years ago. Hypertrophic cardiomyopathy is characterised by left ventricular hypertrophy, which is more often asymetric and mainly involves the interventricular septum. The aim is to demonstrate that hypertrophic cardiomyopathy is a cardiac condition with many unknown features, including definitive confirmation of acute coronary syndrome.

Methods: Clinical examination, laboratory diagnostics, ECG, Doppler ultrasound, coronary arteriography.

Case Report: A 40-year old woman without significant cardiovascular risk factors was admitted to the coronary care unit with the diagnosis of acute coronary syndrome without ST-segment elevation (NSTEMI). Chest pain began two hours before the admission and was preceded by an emotional stress (loss of job) and abuse of large amount of alcohol. The patient had asymmetric hypertrophic cardiomyopathy and has took large amounts of alcohol on weekends throughout the whole last yea. Blood pressure at admission was 150/90 mmHg, heart rhythm was regular and accompanied by increased heart rate, and there was a pansystolic murmur over the base of the heart. Findings on lungs were normal and leg edema was absent. SE, CBC, thyroid status, and kidney function parameters were normal. CK was 1023 $\mathrm{U} / \mathrm{L}$, and the level of troponin I was $5.6 \mathrm{ng} / \mathrm{mL}$. ECG showed sinus rhythm, frequency 130 /minute, PR of $0.20 \mathrm{sec}$, narrow Q wave in D1, D2, D3, aVL, aVF, and V5-6, and deeply negative $\mathrm{T}$ waves in $\mathrm{V} 1-4$ that were in significant regression at discharge. Coronarography performed on the third day of hospitalization showed completely normal results.

Conclusion: In our opinion the fast heart rate increased pressure gradient in the left ventricle outlet which contributed to a decrease in coronary perfusion in spite of normal condition of the coronary arteries. Coronary vasospasm that caused NSTEMI in settings of increased heart rate and massive ventricular hypertrophy as well as can metabolic changes in microcirculation as part of hypertrophic cardiomyopathy cannot be ruled out. The reversibility of vasospasm might be a part of complex reasons for normal coronarography result that was contributed by younger age, female sex, and absence of cardiovascular risk factors. A normal coronary angiogram does not necessarily mean the absence of any atherosclerosis.

KEYWORDS: acute coronary syndrome, coronarography, hypertrophic cardiomyopathy.

CITATION: Cardiol Croat. 2013;8(9):282.

\section{Received: $5^{\text {th }}$ Jul 2013}

*Address for correspondence: The Faculty of Medical Science University of Pristina, Anri Dinana bb, 38220 Kosovska Mitrovica, Kosovo.

Phone: +381666060569

E-mail: snezana_lazic@yahoo.com

\section{Literature}

1. Bugiardini R, Bairey Merz CN. Angina with "normal" coronary arteries: a changing philosophy. JAMA. 2005;293(4):477-84. 